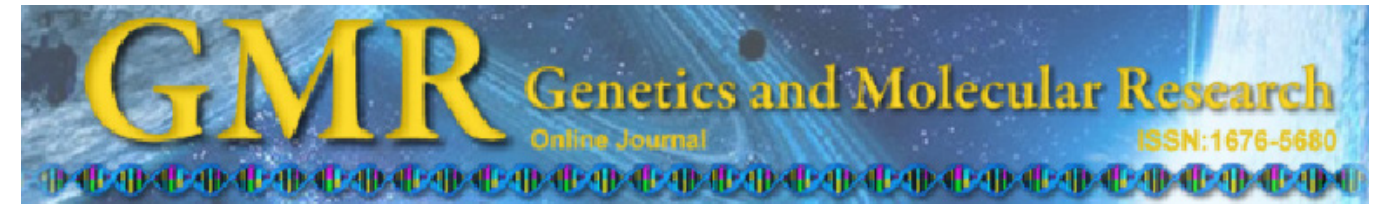

\title{
5-Aza-2'-deoxycytidine may influence the proliferation and apoptosis of cervical cancer cells via demethylation in a dose- and time-dependent manner
}

\author{
T.T. Yao ${ }^{1,2 *}$, S.M. Mo ${ }^{3 *}$, L.Y. Liu ${ }^{1}$, H.W. Lu' ${ }^{1}$, M.L. Huang ${ }^{1}$ and Z.Q. Lin ${ }^{1}$ \\ ${ }^{1}$ Department of Gynecological Oncology, Sun Yat-sen Memorial Hospital, \\ Sun Yat-sen University, Guangzhou, P.R. China \\ ${ }^{2}$ Key Laboratory of Malignant Tumor Gene Regulation and Target Therapy, \\ Guangdong Higher Education Institutes, Sun Yat-sen University, \\ Guangdong, P.R. China \\ ${ }^{3}$ Department of Gynaecology and Obstetrics, Kiang Wu Hospital, \\ Macau, P.R. China \\ *These authors contributed equally to this study. \\ Corresponding author: Z.Q. Lin \\ E-mail: linzhongqiu@hotmail.com
}

Genet. Mol. Res. 12 (1): 312-318 (2013)

Received September 11, 2012

Accepted January 7, 2012

Published February 4, 2013

DOI http://dx.doi.org/10.4238/2013.February.4.5

\begin{abstract}
The methylation of tumor suppressor genes has been shown to be involved in many human cancers. 5-Aza-2'-deoxycytidine (5-Aza$\mathrm{CdR}$ ) can reactivate the expression of methylated tumor suppressor genes. In our study, 2 human cervical cancer cell lines, HeLa and SiHa, were treated with different concentrations $(20,10,5$, and $2.5 \mu \mathrm{M})$ of 5 -Aza-CdR for 24,48 , and $72 \mathrm{~h}$. After incubation, cells were analyzed by 3-(4,5-dimethyl-2-thiazolyl)-2,5-diphenyl-2H-tetrazolium bromide assay and flow cytometry. The expression of RASSF $1 A$ and APAF1 was detected by RT-PCR. 5-Aza-CdR inhibited the growth of HeLa and $\mathrm{SiHa}$ cells at different concentrations. The strongest inhibition and apoptosis rates were obtained after incubation for $72 \mathrm{~h}(5.63 \pm 1.38$ and
\end{abstract}


$8.24 \pm 2.40 \%$, respectively). No significant difference in the expression of $R A S S F 1 A$ was found upon drug treatment, while $A P A F-1$ expression increased in HeLa cells after treatment $(0.790 \pm 0.056 \%)$. Our results suggest that the tumor-suppressive effect of 5-Aza-CdR may result from the reactivation of silenced $A P A F-1$ through demethylation.

Key words: 5-Aza-2'-deoxycytidine; Cervical cancer; $A P A F-1$; DNA methylation; RASSF1A

\section{INTRODUCTION}

Cervical cancer is the second most common cancer in women worldwide (Sankaranarayanan and Ferlay, 2006). Recent studies have revealed the genetic and epigenetic changes involved in cellular malignancy. Epigenetic changes modify the expression of genes without altering their DNA sequences. DNA methylation is the most common DNA modification in eukaryocytes. The hypermethylation of tumor suppressor gene promoters inhibits their transcription and inactivates these genes. Aberrant tumor suppressor gene methylation has been identified in many human cancers (Montenegro et al., 2012; Ozdemir et al., 2012; Menschikowski et al., 2012).

Ras association domain family $1 \mathrm{~A}(R A S S F 1 A)$ is a tumor suppressor gene that has been observed to be highly methylated in many malignant human tumors. Apoptotic protease activating factor-1 (APAF-1), the human analog of the Caenorhabditis elegans ced-4 gene, is a critical factor in activating the caspase cascade. Inactivation of $A P A F-1$ may be due to hypermethylation of several genes.

5-Aza-2'-deoxycytidine (5-Aza-CdR) is a pyrimidine nucleoside analog that can integrate into DNA and block methylation by forming a covalent complex with DNA methyltransferase. It can reactivate the expression of methylated tumor suppressor genes and restore their anti-tumorigenic activities (Hassler et al., 2012; Chu et al., 2012). In this study, we investigated the effects of 5-Aza$\mathrm{CdR}$ on cervical carcinoma cells in an attempt to provide new insights into cervical cancer treatment.

\section{MATERIAL AND METHODS}

\section{Cell culture}

The human cervical adenocarcinoma cell line HeLa and squamous cancer cell line SiHa were purchased from American Type Culture Collection and maintained in RPMI 1640 medium (Life Technologies, USA) supplemented with 10\% fetal bovine serum (Invitrogen, USA). They were treated with 5-Aza-CdR (Sigma, USA) at different concentrations $(2.5,5$, 10 , and $20 \mu \mathrm{M}$ ) for 24,48 , and $72 \mathrm{~h}$.

\section{3-(4,5-Dimethyl-2-thiazolyl)-2,5-diphenyl-2H-tetrazolium bromide (MTT) assay}

Cell survival was assessed using the MTT assay (Sigma). MTT was added at a final concentration of $0.5 \mathrm{mg} / \mathrm{mL} 72 \mathrm{~h}$ after chemical intervention with 5-Aza-CdR, and the cells were incubated for $4 \mathrm{~h}$ at $37^{\circ} \mathrm{C}$ and dissolved in $150 \mu \mathrm{L}$ DMSO (Sigma). Optical density values were read at $570 \mathrm{~nm}\left(\mathrm{~A}_{570}\right)$ using an Easy Reader 340 AT (EAR400 SLT-Lab. Instruments, Research 
Triangle Park, NC, USA). Each experiment was carried out in triplicate and repeated 3 times.

\section{Annexin-V analysis}

Cell pellets were resuspended in Annexin-V-FLUOS staining solution (LightCycler, Roche Molecular Biochemicals, Mannheim, Germany) and incubated for $15 \mathrm{~min}$ at room temperature. Samples were then analyzed on an FSCAN flow cytometer (Hershey Medical Center Core Facility).

\section{Reverse transcriptase-polymerase chain reaction (RT-PCR)}

Total RNA was extracted using the TRIzol reagent (Invitrogen), and RT-PCR was performed using the PrimerScript RT-PCR kit (TaKaRa Biotechnology, China) according to manufacturer instructions on the DNA Engine Peltier Thermal Cycler (PTC-200, Bio-Rad Laboratories, Inc., China) with specific primers.

Primer sequences for detection were as follows: RASSF1A - forward: 5'-TTGGGTGAC CTCTTGTACCC-3', reverse: 5'-TGGCACTGTAGAGAGAAACCAA-3', and APAF-1 - forward: 5'-TTGCTGCCCTTCTCCATGAT-3', reverse: 5'-TCCCAACTGAAACCCAATGC-3'. $\beta$-actin (forward:5'-GTCCACCTTCCAGCAGATGT-3', reverse:5'-CACCTTCACCGTTCCAGTTT$3^{\prime}$ ) was used as an endogenous control. These primers yielded 497-, 285-, and 245-bp products, respectively.

The PCR cycling conditions were as follows: initial hold at $65^{\circ} \mathrm{C}$ for $5 \mathrm{~min}, 42^{\circ} \mathrm{C}$ for $30 \mathrm{~min}$ for cDNA synthesis, followed by amplification for 30 cycles at $95^{\circ} \mathrm{C}$ for $30 \mathrm{~s}, 55^{\circ} \mathrm{C}$ for $30 \mathrm{~s}$, and $72^{\circ} \mathrm{C}$ for $1 \mathrm{~min}$. All reactions were performed in triplicate, and a negative control lacking cDNA was included. PCR products were then separated on $1.5 \%$ agarose gels containing ethidium bromide and visualized by UV transillumination.

\section{Statistical analysis}

All statistical analyses were carried out using the SPSS for Windows version 13.0 software. All results are reported as means \pm SE from at least 3 separate experiments. The differences between groups were analyzed using the Student $t$-test when only 2 groups were compared or by one-way analysis of variance for all other analyses. All tests were 2-sided. Differences were considered to be statistically significant at $\mathrm{P}<0.05$.

\section{RESULTS}

The effects of 5-Aza-CdR derivatives on DNA methylation and the expression of $R A S S F 1 A$ and $A P A F-1$ were examined after exposure at different concentrations $(20,10,5$, $2.5 \mu \mathrm{M})$ for 24,48 , and $72 \mathrm{~h}$.

\section{Cell proliferation}

Cell proliferation was suppressed in a concentration-dependent manner in HeLa cells (Figure 1A). The $20 \mu \mathrm{M}$ treatment group showed increasing growth inhibition rates with time. 
The other 3 treatment groups reached maximum growth inhibition rates after exposure for 48 $\mathrm{h}$, followed by a slight decrease from 48 to $72 \mathrm{~h}$. These findings suggest that a high 5-Aza-CdR concentration may more effectively suppress HeLa growth. Compared to treatment with other doses, the inhibition rate was lower in SiHa cells upon treatment with $2.5 \mu \mathrm{M}$ drug (Figure 1B). These results suggest that there may be an optimum dose for suppressing tumor cell proliferation. After $72 \mathrm{~h}$, all $\mathrm{SiHa}$ groups showed similar inhibition rates, suggesting that a low-dose treatment could achieve optimal inhibition by prolonging treatment course.

A

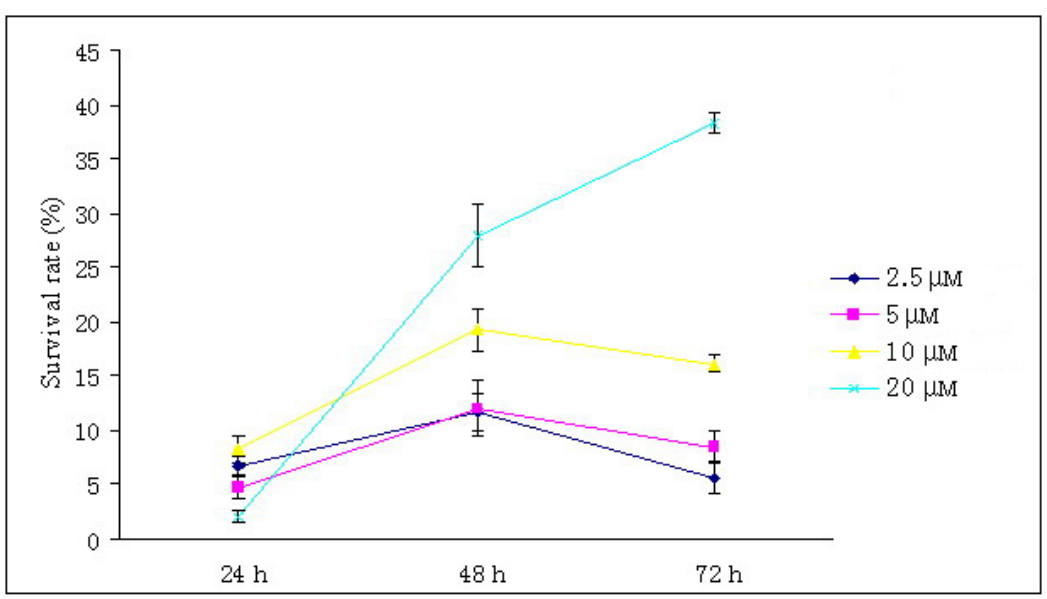

B

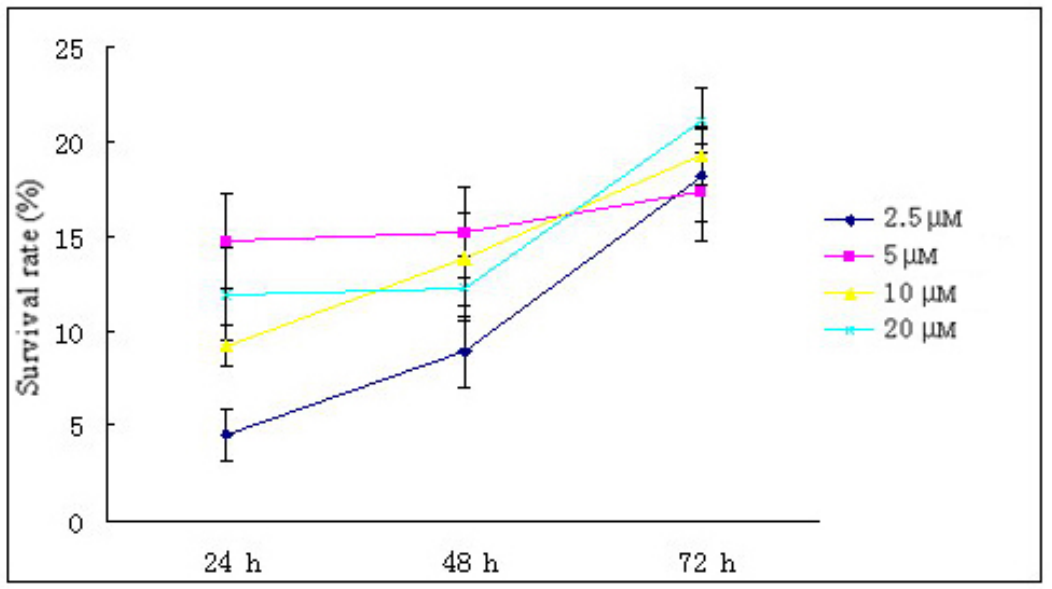

Figure 1. Growth inhibitory after exposure to 5-Aza-CdR. A. HeLa; B. SiHa.

\section{Cell apoptosis}

The apoptosis rate of HeLa cells increased significantly after exposure to $5 \mu \mathrm{M} 5$-Aza$\mathrm{CdR}$ for $72 \mathrm{~h}$ as determined by flow cytometric analysis (Figure 2), while the apoptosis rate of $\mathrm{SiHa}$ cells increased significantly upon treatment with $20 \mu \mathrm{M}$ 5-Aza-CdR. 


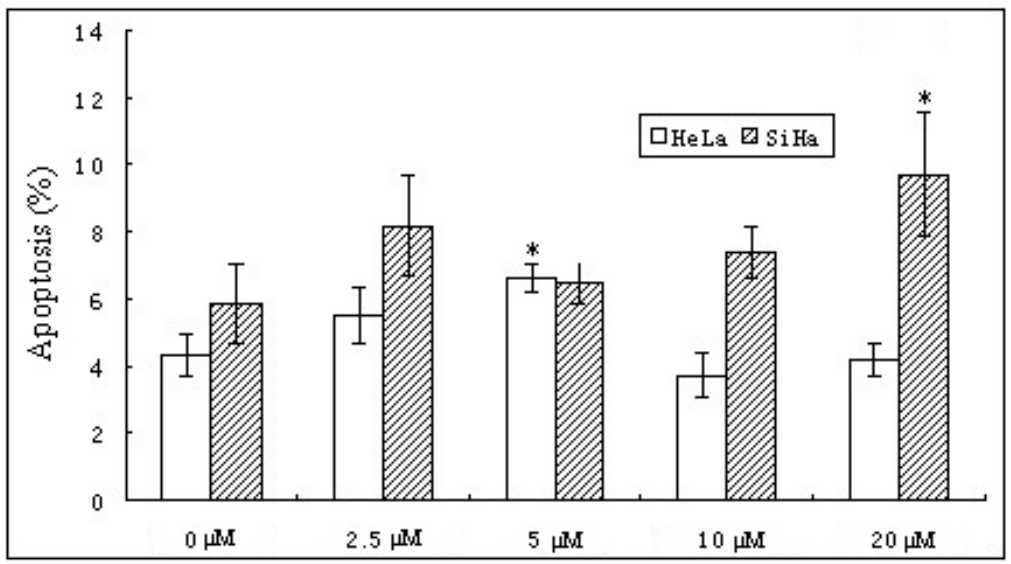

Figure 2. Flow cytometric analysis results.

\section{$R A S S F 1 A$ and $A P A F-1$ expression}

The expression of RASSF1A and APAF-1 mRNA could be detected in HeLa and $\mathrm{SiHa}$ cells before 5-Aza-CdR treatment. After treatment with 5-Aza-CdR, the expression of RASSF1A did not change significantly. The expression of APAF-1 mRNA increased in HeLa cells after treatment with $20 \mu \mathrm{M} 5$-Aza-CdR, while its expression was not significantly different in SiHa cells before and after treatment with 5-Aza-CdR (Figure 3A and B).

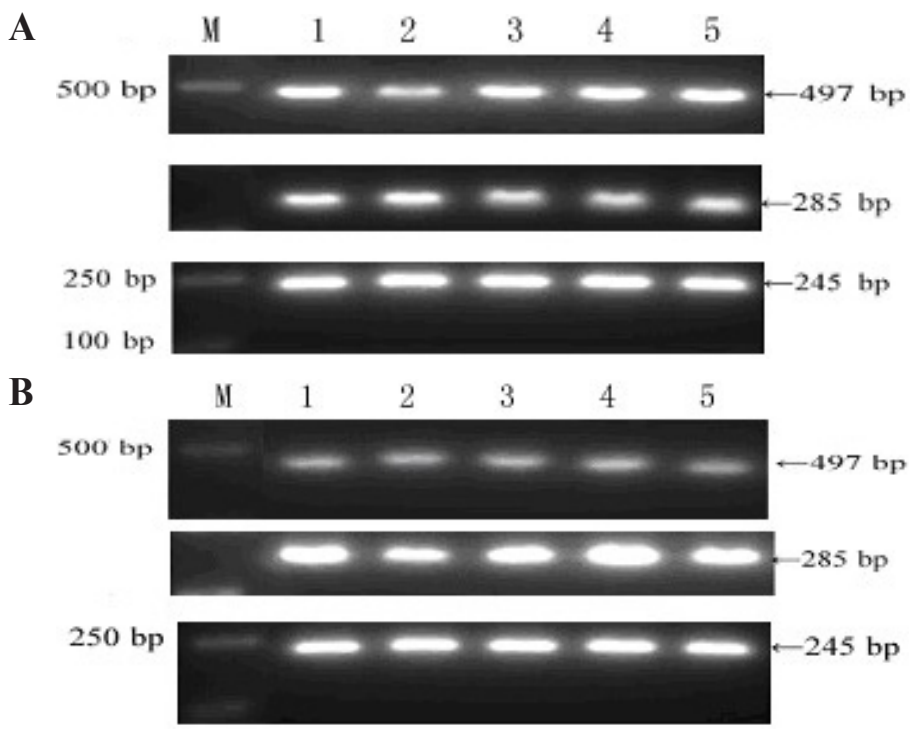

Figure 3. Expression of RASSF1A and APAF-1 mRNA by RT-PCR. A. HeLa; B. SiHa. Lane M= molecular maker 2000; lane $1=20 \mu \mathrm{M}$ 5-Aza-CdR; lane $2=10 \mu \mathrm{M}$ 5-Aza-CdR; lane $3=5 \mu \mathrm{M}$ 5-Aza-CdR; lane $4=2.5 \mu \mathrm{M}$ 5-Aza$\mathrm{CdR}$; lane $5=0 \mu \mathrm{M}$ 5-Aza-CdR. 


\section{DISCUSSION}

5-Aza-CdR is a pyrimidine nucleoside analog that forms a covalent complex with DNMT during DNA replication. No heritable growth inhibition was found treated with varying doses of arabinofuranosyl cytidine, which does not inhibit DNA methylation(Wu et al., 2006). In comparison, after treatment with 5-Aza-CdR, methylation-silenced growth-regulatory genes were reactivated and cervical cells CaSki, C-33A, HeLa, and SiHa showed heritable growth inhibition, demonstrating that 5 -Aza-CdR inhibits tumor cell proliferation by demethylating and reactivating genes silenced by methylation (Wu et al., 2006). We hypothesized that 5-AzaCdR may suppress cervical carcinoma cell proliferation and induce cell apoptosis by changing the methylation status of some genes.

$R A S S F 1 A$ is located on human chromosome 3 at $\mathrm{p} 21.3$. The RASSF1A promoter has been reported to be hypermethylated in $10 \%$ of squamous cell carcinomas, in $21 \%$ of adenosquamous carcinomas, and in $24 \%$ of cervical adenocarcinomas. Promoter hypermethylation has been discovered in various human tumors, including small cell lung, breast, bladder, prostate, gastric, and renal cell carcinomas (Ferguson et al., 1997; Dammann et al., 2001; Dreijerink et al., 2001; Lee et al., 2001; Burbee et al., 2001; Kwong et al., 2002; Kuzmin et al., 2002; Liu et al., 2002). Vos et al. (2000) suggested that RASSF1 binds Ras in a GTP-dependent manner. Kuzmin et al. (2003) observed the methylation of the RASSF $1 A$ promoter in $6 \%$ of normal cervical tissues and in $10 \%$ of squamous carcinomas, $21 \%$ of adenosquamous carcinomas, and $24 \%$ of cervical adenocarcinomas. Moreover, Kuzmin et al. (2003) found that RASSF1A mRNA was expressed in 6 HPV-positive cervical carcinoma cell lines (ME 180, MS751, SiHa, C-4I, HeLa, and CaSki) whereas it was not expressed in 2 HPV-negative cell lines (C-33A and HT-3). This is most likely because HPV infection reduces RASSF $1 A$ inactivation. Consistent with this, our analyses showed that there was no increase in RASSF 1A mRNA levels in HeLa or SiHa cells treated with 5-Aza-CdR.

$A P A F-1$ is located on human chromosome 12q23. Its hypermethylation-based silencing has been confirmed in various malignant human tumors. This hypermethylation can be reversed with demethylating agents (Christoph et al., 2006). In our study, 5-Aza-CdR-treated HeLa cells showed a higher apoptosis rate than untreated HeLa cells. The expression of APAF1 is correlated with cancer cell apoptosis (Andreev et al., 2012; Niimi et al., 2012; Melzer et al., 2012). Presumably, 5-Aza-CdR reduces $A P A F-1$ methylation and restores its expression, thus reactivating its functions. In comparison, $A P A F-1$ gene expression in $\mathrm{SiHa}$ cells appeared unchanged after treatment. Whether $A P A F-1$ expression is related to HPV infection needs to be clarified by future studies.

In summary, the present study shows that 5-Aza-CdR can suppress in vitro proliferation of cervical carcinoma cells and induce their apoptosis. These results provide experimental support for and new insights into novel therapies for cervical cancer. However, the present study is limited to in vitro experiments. Therefore, further studies will be necessary to better understand the effects of 5-Aza-CdR on cervical cancer.

\section{Conflict of interest}

The authors declare no conflict of interest. 


\section{ACKNOWLEDGMENTS}

Research supported by the National Natural Science Foundation of China (\#81101979), the Guangdong Province Natural Scientific Grant (\#S2011040004639), the Guangdong Province Medical Science Technology Grant (\#B2011088), the Key Clinical Program of the Ministry of Health [(2010) 439], the Yat-sen Scholarship for Young Scientists, and the Program for Training Young Scientists in Sun Yat-sen University.

\section{REFERENCES}

Andreev DE, Dmitriev SE, Zinovkin R, Terenin IM, et al. (2012). The 5' untranslated region of Apaf-1 mRNA directs translation under apoptosis conditions via a 5' end-dependent scanning mechanism. FEBS Lett. 586: 4139-4143.

Burbee DG, Forgacs E, Zochbauer-Muller S, Shivakumar L, et al. (2001). Epigenetic inactivation of RASSF1A in lung and breast cancers and malignant phenotype suppression. J. Natl. Cancer Inst. 93: 691-699.

Christoph F, Kempkensteffen C, Weikert S, Kollermann J, et al. (2006). Methylation of tumour suppressor genes APAF-1 and DAPK-1 and in vitro effects of demethylating agents in bladder and kidney cancer. Br. J. Cancer 95: 1701-1707.

Chu SH, Ma YB, Feng DF, Zhang H, et al. (2012). Effect of 5-Aza-2'-deoxycytidine on SLC22A18 in glioma U251 cells. Mol. Med. Report. 5: 138-141.

Dammann R, Takahashi T and Pfeifer GP (2001). The CpG island of the novel tumor suppressor gene RASSF1A is intensely methylated in primary small cell lung carcinomas. Oncogene 20: 3563-3567.

Dreijerink K, Braga E, Kuzmin I, Geil L, et al. (2001). The candidate tumor suppressor gene, RASSF1A, from human chromosome 3p21.3 is involved in kidney tumorigenesis. Proc. Natl. Acad. Sci. U. S. A. 98: 7504-7509.

Ferguson AT, Vertino PM, Spitzner JR, Baylin SB, et al. (1997). Role of estrogen receptor gene demethylation and DNA methyltransferase.DNA adduct formation in 5-aza-2'deoxycytidine-induced cytotoxicity in human breast cancer cells. J. Biol. Chem. 272: 32260-32266.

Hassler MR, Klisaroska A, Kollmann K, Steiner I, et al. (2012). Antineoplastic activity of the DNA methyltransferase inhibitor 5-aza-2'-deoxycytidine in anaplastic large cell lymphoma. Biochimie 94: 2297-2307.

Kuzmin I, Gillespie JW, Protopopov A, Geil L, et al. (2002). The RASSF1A tumor suppressor gene is inactivated in prostate tumors and suppresses growth of prostate carcinoma cells. Cancer Res. 62: 3498-3502.

Kuzmin I, Liu L, Dammann R, Geil L, et al. (2003). Inactivation of RAS association domain family 1A gene in cervical carcinomas and the role of human papillomavirus infection. Cancer Res. 63: 1888-1893.

Kwong J, Lo KW, To KF, Teo PML, et al. (2002). Promoter hypermethylation of multiple genes in nasopharyngeal carcinoma. Clin. Cancer Res. 8: 131-137.

Lee MG, Kim HY, Byun DS, Lee SJ, et al. (2001). Frequent epigenetic inactivation of RASSF1A in human bladder carcinoma. Cancer Res. 61: 6688-6692.

Liu L, Yoon JH, Dammann R and Pfeifer GP (2002). Frequent hypermethylation of the RASSF1A gene in prostate cancer. Oncogene 21: 6835-6840.

Melzer IM, Fernandez SB, Bosser S, Lohrig K, et al. (2012). The Apaf-1-binding protein Aven is cleaved by Cathepsin D to unleash its anti-apoptotic potential. Cell Death. Differ. 19: 1435-1445.

Menschikowski M, Platzbecker U, Hagelgans A, Vogel M, et al. (2012). Aberrant methylation of the M-type phospholipase A2 receptor gene in leukemic cells. BMC Cancer 12: 576.

Montenegro MF, Saez-Ayala M, Pinero-Madrona A, Cabezas-Herrera J, et al. (2012). Reactivation of the Tumour Suppressor RASSF1A in Breast Cancer by Simultaneous Targeting of DNA and E2F1 Methylation. PLoS One 7: e52231.

Niimi S, Arakawa-Takeuchi S, Uranbileg B, Park JH, et al. (2012). Cdc6 protein obstructs apoptosome assembly and consequent cell death by forming stable complexes with activated Apaf-1 molecules. J. Biol. Chem. 287: 18573-18583.

Ozdemir F, Altinisik J, Karateke A, Coksuer H, et al. (2012). Methylation of tumor suppressor genes in ovarian cancer. Exp. Ther. Med. 4: 1092-1096.

Sankaranarayanan R and Ferlay J (2006). Worldwide burden of gynaecological cancer: the size of the problem. Best. Pract. Res. Clin. Obstet. Gynaecol. 20: 207-225.

Vos MD, Ellis CA, Bell A, Birrer MJ, et al. (2000). Ras uses the novel tumor suppressor RASSF1 as an effector to mediate apoptosis. J. Biol. Chem. 275: 35669-35672.

Wu Y, Meng L, Wang H, Xu Q, et al. (2006). Regulation of DNA methylation on the expression of the FHIT gene contributes to cervical carcinoma cell tumorigenesis. Oncol. Rep. 16: 625-629. 